\title{
El turismo rural y aportaciones al desarrollo de comunidades en territorios locales
}

\section{Rural tourism and contributions to the development of communities in territories local}

Alexandra Verónica Parra Cárdenas. ${ }^{1}$, Lourdes Cisneros Mustelier. ${ }^{2}$ \& Efraín Velasteguí López. ${ }^{3}$

\begin{abstract}
.
Rural tourism, an activity of a territorial nature, whose importance is ratified in the present century by the wear and tear of conventional tourism offers, the emergence of environmental awareness and thinking, the need to create innovative ways of life for communities in states of poverty and entitled to claim. Tourism that executed in an optimal state helps to preserve the natural and cultural resources of the destination communities, while creating competitive advantages, generating sources of work and income from foreign currency. The purpose of this article is to present a vision of rural tourism through of two axes: a theoretical-conceptual and a practical one related to the Ecuadorian community rural tourism activity, a documentary review is presented that integrates more relevant information on this topic in question. The results yielded important conclusions that show the importance of conducting research studies that provide effective solutions to problems of tourism activities in rural territories is $30.5 \%$
\end{abstract}

Keywords: Rural tourism, competitiveness, community, territory, activity tourist.

\section{Resumen.}

El turismo rural, actividad de carácter territorial, cuya importancia se ratifica en el presente siglo por el desgaste de ofertas de turismo convencional, el surgimiento de una consciencia y pensamiento ecologista, la necesidad de crear formas innovadoras de vida para comunidades en estados de pobreza y con derecho a la reivindicación. Turismo que ejecutado en un estado óptimo contribuye a preservar los recursos naturales y culturales de las comunidades de destino, mientras se va creando ventajas competitivas, generando fuentes de trabajo e ingresos de divisas El

\footnotetext{
${ }^{1}$ Universidad de las Fuerzas Armadas ESPE, Departamento de Ciencias Económicas, Administrativas y de Comercio CEAC, Sangolquí, Ecuador, avparra@espe.edu.ec

${ }^{2}$ Universidad de La Habana, Cuba, Facultad de Turismo, Cuba, lourdes_cisneros@ftur.uh.cu

${ }^{3}$ Ciencia Digital, Ambato, Ecuador, luisefrainvelastegui @ cienciadigital.org
} 
propósito del presente artículo es presentar una visión del turismo rural a través de dos ejes: una teórico-conceptual y una práctico relacionado con la actividad turística rural comunitaria ecuatoriana, se presenta una revisión documental que integra información más relevante de esta temática en mención. Los resultados arrojaron importantes conclusiones que evidencian la importancia de realizar estudios investigativos que den soluciones efectivas a problemas de actividades turísticas en territorios rurales es de $30.5 \%$.

Palabras claves: Turismo rural, competitividad, comunitario, territorio, actividad turística.

\section{Introducción}

Una nueva visión global del mundo rural surge desde hace más de dos décadas, pues diferentes investigaciones ponen de manifiesto los cambios demográficos, socioeconómicos, culturales, políticos en espacios rurales de Estados Unidos y Europa Occidental, cuyas características estaban relacionadas con el auge demográfico en estos territorios, detectándose a la vez no solamente la desagrarización, también lo es, el surgimiento de nuevas actividades, relacionadas entre otras, con el sector de servicios, una mejora de su infraestructura y mejores niveles de la calidad de vida de la población, hablándose del resurgimiento de las zonas rurales (Kayser, 1990).

En Latinoamérica y El Caribe, un importante volumen de comunidades rurales ha integrado en sus dinámicas sociales, productivas y económicas, actividades turísticas como una alternativa para luchar contra la pobreza, incorporando a los habitantes, que en oportunidades son grupos de indígenas y mestizos, generando una nueva forma de hacer Turismo y que ha sido denominado Turismo Rural Comunitario. En tal sentido, autores como Gascón (2009) citado por Kieffer (2018), indica que el turismo rural comunitario es un tipo de Turismo implementado y desplegado en espacios rurales donde los habitantes locales organizados colectivamente, juegan un rol participativo en su progreso, gestión y monitoreo, procurando el respeto por el entorno natural, sociocultural y los valores ancestrales de su comunidad, permitiendo un disfrute agradable, favorable y reciproco de experiencias entre los pobladores locales y los turistas (visitantes), en la cual esta interrelación turista-comunidad rural, debiera ser caracteriza por la justicia en la distribución equitativa de los beneficios generados por la ejecución.

Para el caso de Latinoamérica la incorporación del turismo a los espacios rurales es observada como una importante actividad económica del sector de los servicios(turismo), por las siguientes razones: es evidente la existencia de relaciones humanas resultantes de la interacción entre turistas

y comunidades campesinas; aprovechando y disfrutando el ambiente, los valores naturales, culturales y socio-productivos, otro beneficio de su práctica es evitar el éxodo de la población de las comunidades, quienes se convierten en actores y protagonistas del turismo, adicionalmente, se ocupan factores ociosos como capital y mano de obra. Constituye una actividad que se autofinancia a través de las oportunidades para el desarrollo de proyectos para el aprovechamiento turístico bajo una óptica de sostenibilidad, siempre como una forma complementaria a la propia estructura local bajo la necesidad de poseer una adecuada política turística. 
En definitiva, siendo el turismo una actividad importante de ingreso de divisas, cuya competitividad requiere de una diversificación en su oferta, aparece el turismo rural como una estrategia fácil y rápida de desarrollo y lucha contra la pobreza, capaz de generar beneficios con muy poca inversión, ya que su principal atracción (el paisaje y la cultura autóctona) ya estaban gratuitamente (Burns \& Holden, 1995; Mowforth \& Munt, 1998; Hamilton, 2006; Hickman, 2007; Nowicka, P, 2008).

\section{Beneficios y dimensiones del turismo en territorio rural}

El turismo es un sector dinámico y generador de desarrollo y específicamente siendo el turismo rural conocido también como comunitario, es aquel que está orientado a promover actividades turísticas con participación local, su demanda crece permanentemente en contraposición al turismo tradicional.

El turismo comunitario en territorios rurales es presentado por primera vez en 1985 por Murphy, quien establece la relación turismo-áreas naturales en países comprometidos económicamente y con índices de pobreza muy evidentes, posteriormente en el año 2004, el mismo autor (Murphy) y en trabajos de los investigadores precedentes Richards y Hall en el año 2000, se presenta a esta modalidad turística como un aliado para combatir y lograr reducir la pobreza. En otras palabras, puede considerarse este tipo de turismo muy adecuado para países en vías de desarrollo, pues es un instrumento esencial para disminuir los niveles de pobreza de las zonas deprimidas económicamente de la región y favorecer el crecimiento de sus economías, mientras se resguarda su patrimonio y valores étnico-cultural-históricos y se fortalece su identidad (Jaime-Pastor, CasasJurado, \& Amparo-Soler, 2011; Maldonado, 2005).

Una de las concepciones más claras es la planteada por el ICT/Alianza para el fortalecimiento del turismo rural comunitario que lo define como "experiencias turísticas planificadas e integradas sosteniblemente al medio rural y desarrollada por los pobladores locales, organizados para los beneficios de la comunidad". En este sentido es la organización comunal quien planifica dicha actividad turística que también posee la característica de actividad económica, siendo los pobladores de estas comunidades quienes participan en forma directa en la gestión de servicios. En su interior se articula el producto turístico, por lo cual, es importante la participación de estos actores locales.

Esta modalidad turística genera beneficios significativos a las áreas rurales, ya que la incidencia es inmediata y directa, primeramente sobre las familias de las localidades, en el cambio favorable de la calidad y estilos de vida y en el desarrollo local, posteriormente el impacto sobre el estímulo de una nueva clase de turismo, el cual es garante de una mejora sustancial de la calidad de vida de regiones rurales disminuyendo los índices de pobreza, preservación de los recursos naturales y culturales de las comunidades-destino, mientras que conjuntamente va creando ventajas competitivas, ingresos de divisas y empleos dignos contextualizados con la realidad local (WWF International, 2001; OMT, Manual on Tourism and poverty alleviation, 2002; Manyara \& Jones , 2007; Bolwel \& Weinz , 2009) 
En diversas oportunidades y circunstancias, se lo ha asimilado al término de Turismo de Naturaleza, en virtud de que la gran parte de sus actividades turísticas se disfrutan en ambientes naturales. También por las múltiples modalidades que ofertan las comunidades rurales se lo ha identificado como: agroturismo, turismo étnico-cultural-religioso-esotérico, turismo deportivoaventura, turismo educativo-técnico-científico-de salud, turismo de eventos, gastronómico, ecoturismo, turismo en comunidades de recreación y retiro, y muy frecuentemente se le considera como una variación o categoría del Turismo Cultural, en el contexto del encuentro con novedosas manifestaciones tradicionales (costumbres) y actividades autóctonas de las localidades rurales, en resumen el Turismo Cultural o se considera una variante del Turismo Rural o éste se considera una de las prácticas del Turismo Cultural (Barrera \& Muñoz, 2003; Toselli, 2003)

La actividad del Turismo generada en los espacios rurales, mayormente se asocia e identifica con procesos de organización comunitaria donde los atractivos turísticos (naturales, culturales, arquitectónicos, entre otros) están considerados como bienes públicos que pertenecen a la comunidad, donde los habitantes locales son los gestores encargados de su aprovechamiento y control y permiten un encuentro activo con los visitantes. El turismo rural comunitario se enfoca en la participación más beneficiosa de los actores y gestores de la localidad en la formulación de las diversas propuestas turísticas, el control óptimo sobre los recursos generados, la justa distribución de los beneficios, mediante la toma de decisiones en un marco de respeto y consenso comunitario que facilite la inclusión e integración de los miembros de la comunidad (Campbell, 1999; Stronza \& Gordillo, 2008; Zizumbo-Villareal, 2013; Pérez, 2004).

A continuación, se presentan la fundamentación sobre las dimensiones de esta actividad turística (Ver cuadro 1):

Cuadro 1. Dimensiones del Turismo Rural

\begin{tabular}{ll}
\hline Dimensiones & Fundamentación de las dimensiones \\
\hline Cultural & La Humanidad inicia su estructura histórica-política-social. El lenguaje, las creencias, la espiritualidad, \\
& ritos y tradiciones heredadas, los saberes aprendidos, las relaciones micro y macro cósmicas, la \\
& comunicación con sus pares, los modos y medios para aprovisionarse de alimentos y resolver su \\
& subsistencia son los atributos y trazas culturales que caracterizan y dan identidad al sector rural. La \\
& percepción externa de tradiciones, instrumentos, historia, artesanías y demás expresiones culturales, crea \\
& en la localidad una confirmación de la identidad y la posibilidad simbólica y real de conservarlas \\
& Los efectos económicos del turismo rural son la generación de ingresos - Divisas, en caso de Turismo \\
Económica & Internacional, los puestos de trabajo necesarios generados para atender la demanda, la mejora del mercado \\
& de producción rural, primaria, industrial y artesanal, visibilizando productos con identidad territorial. \\
Social & El turismo rural impulsa, colabora y favorece la inclusión de jóvenes y mujeres en la actividad laboral, y \\
& mediante el empleo, favorece el arraigo rural. Las oportunidades para sectores marginados se incrementan \\
& cuando se trata de modalidades como el turismo comunitario. Se favorece el emprendimiento agropecuario \\
& y las posadas turísticas rurales, \\
\hline
\end{tabular}


Ambiental

Política
El turismo rural se considera de bajo impacto ambiental, sus beneficios pueden resumirse por el mantenimiento, preservación y mejora de los espacios naturales, y los costos, por el riesgo de aumento de la contaminación, perturbación de la flora y fauna y la erosión del suelo

Desde la esfera rural, la ordenación del territorio está relacionada con políticas públicas intervencionistas

del espacio rural en función de un proyecto político nacional

Fuente: (Varisco C. , 2016)

Considerando lo expuesto los autores afirman que definitivamente el turismo bien planificado, ejecutado y evaluado constituye un instrumento de desarrollo y requiere la participación de varios actores siendo el principal la misma comunidad, según (López-Cordonez , 2017) el turismo rural comunitario está contextualizado por la autogestión y control de los beneficios sociales y económicos realizados de forma directa por la comunidad, valorando sus tradiciones y culturas ancestrales, así como los recursos naturales del entorno, promoviendo el orgullo por la identidad local, la pertenencia y la interculturalidad y bajo la esfera de políticas públicas que lo apoyen. Por tanto, el desarrollo de las comunidades cobra la relevancia necesaria para lograr la movilización de personas hacia espacios que le ofrezcan la oportunidad de experiencias únicas y novedosas para estar en contacto directo con el medio ambiente y el intercambio con culturas y costumbres diferentes.

\section{Experiencias sobre turismo rural en Ecuador}

Los países andinos poseen características únicas para el despliegue de este nuevo tipo de turismo que se desarrolla en los sectores rurales, pues el cambio en gustos y preferencias, la revalorización de lo tradicional, un interés por el medio ambiente, así como los beneficios que se derivan de esta, provocan un permanente interés por mejorar la oferta y ser competitivos.

Ecuador es un país andino que cuenta con una amplia biodiversidad y riqueza cultural, con cuatro regiones bien diferenciadas, son el asiento de diversidad de flora y fauna, las cuales son evidentes en las 26 áreas bajo régimen de protección por parte del Estado. De la misma forma su atractivo patrimonio histórico y tradicional, así como el gastronómico, el cultural, ha favorecido diversas formas de Turismo, entre ellas en territorio Rural, el cual se ha afianzado como una táctica para el desarrollo de las comunidades, así como objeto de amplias investigaciones, En tal sentido, concentra tres aspectos esenciales: el hábitat natural y las manifestaciones culturales; la sostenibilidad natural y social y el monitoreo del turismo por parte de las localidades. El Turismo Rural se asocia como uno de los contribuyentes a la marca-país internacionalmente mediante un incremento en la oferta y demanda turística (Zurita, Román, Fonseca, Quevedo, \& Castillo, 2017).

Hacia finales en 1950 Ecuador marco políticas modernizantes qué propone al turismo como instrumento de desarrollo para el país. Estos esfuerzos iníciales tuvieron logros modestos en atraer turistas y la consolidación de la infraestructura y negocios turísticos; el país ha sido y sigue siendo una estación de turismo de pequeña y mediana intensidad, con evidencias en los últimos años del crecimiento de la actividad. Hacía 1995 se hizo la primera recopilación sobre ecoturismo en Ecuador mediante la evaluación de experiencias desarrolladas en el marco de un programa de 
protección de bosques oficiado por agencias de conservación. El análisis de las experiencias mostró resultados mixtos y evidenciaron que el ecoturismo no resolvería los problemas ambientales. (Prieto, 2011)

Recientemente, el gobierno de Ecuador ha promovido y estimulado al turismo rural comunitario desde la Planificación General, considerándola una actividad provechosa económicamente y a su vez en un sector estratégico del país evidenciando esta realidad en la Agenda de Transformación Productiva del país y El Plan Nacional Toda una Vida donde se vincula al turismo rural comunitario en sus objetivos, plantea favorecer la igualdad, la inclusión y participación en un marco de equidad social y territorial para mejorar la calidad de vida del pueblo por medio de la actividad y disfrute del Turismo como una actividad económica alterna.

Las empresas ecuatorianas han respondido positivamente al reto de las nuevas tendencias de la demanda turística internacional de programas alternativos y especializados. La oferta de actividades deportivas, tales como pesca, rafting, buceo, montañismo y ciclismo, se ha incrementado en un 28\%; mientras que la oferta de turismo de naturaleza especializado, como ecoturismo, observación de flora y fauna, agroturismo, etc. ha tenido un incremento del $66 \%$. (PLANDETUR 2020, 2012),

Con estas proyecciones y considerando que un elemento importante de la actual estrategia de desarrollo económico y social de la región es la transformación de las estructuras productivas con énfasis en áreas que sin ser determinantes en el actual contexto de la globalización contribuyen en forma precisa a resolver dos de los problemas más críticos como son el desempleo y la pobreza, se plantea la promoción del "turismo rural" como alternativa competitiva de mejora para los sectores más desprotegidos (García, 1998)

A continuación, se exponen ejemplos de actividades que fortalecen el turismo rural y otros más refuerzan tácitamente las virtudes del turismo en territorios rurales con una amplia perspectiva de sostenibilidad.

\section{Cuadro 2. Proyectos Turísticos}

\begin{tabular}{llll}
\hline Proyecto & Descripción & Características \\
\hline Ruta del Café & Finca cafetalera en donde & - & Jipijapa o "la Sultana del Café" llamada así \\
Jipijapa & se lleva a cabo un proyecto & & por la próspera y tradicional producción \\
& de Turismo Rural, & cafetalera. \\
denominado la Ruta del & - & La gastronomía del sector es reconocida \\
& Café, que forma parte del & por el famoso "ceviche de maní" y el \\
& proyecto & de & "greñoso". \\
& $\begin{array}{l}\text { Fortalecimiento } \\
\text { Institucional y grupos de }\end{array}$ & En la comuna de Sancán las exquisitas \\
& tortillas de maíz y yuca. \\
\hline
\end{tabular}




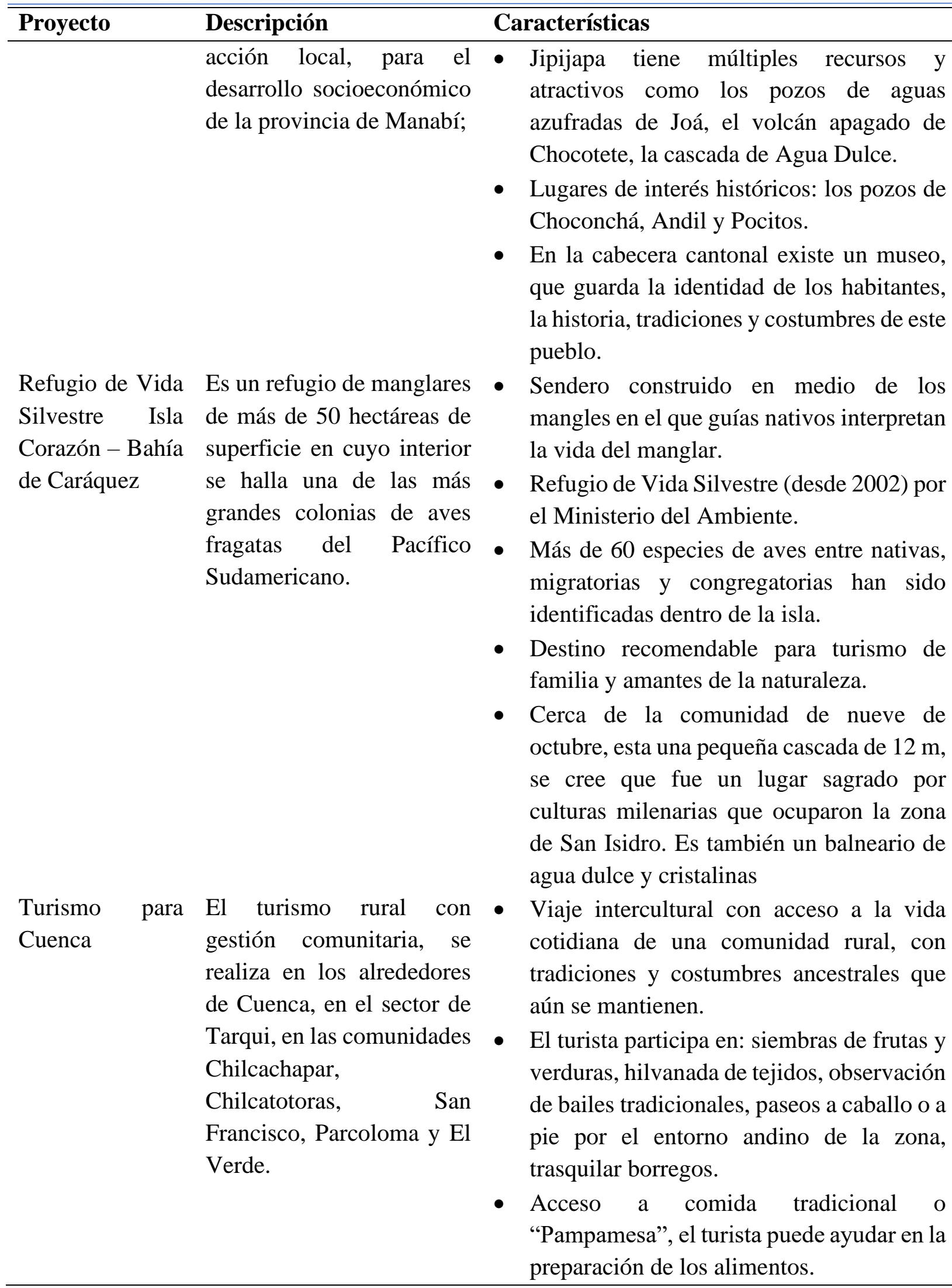




\begin{tabular}{|c|c|c|}
\hline Proyecto & Descripción & Características \\
\hline $\begin{array}{l}\text { Ruta del Valle } \\
\text { del Chota }\end{array}$ & $\begin{array}{l}\text { El Valle del Chota se } \\
\text { encuentra situado entre el } \\
\text { límite de las provincias de } \\
\text { Carchi e Imbabura, a } 35 \\
\text { Km. de Ibarra y } 89 \mathrm{Km} \text {. de } \\
\text { Tulcán, se accede al valle } \\
\text { fácilmente por la } \\
\text { Panamericana Norte, } \\
\text { mismo que está ubicado a } \\
1560 \text { m.s.n.m., tiene una } \\
\text { temperatura promedio de } \\
24^{\circ} \mathrm{C} \text {. }\end{array}$ & $\begin{array}{l}\text { - Una amplia gama como la recreación en } \\
\text { piscinas, canchas deportivas, paseos en } \\
\text { chiva, rafting y kayaking, también están las } \\
\text { artesanías y gastronomía típica. } \\
\text { - El río Chota-Mira se lo conoció como } \\
\text { 'Coangue' y actualmente es un espacio } \\
\text { ideal para la práctica de deportes de } \\
\text { aventura. } \\
\text { - Elaboran varios productos como vinos y } \\
\text { mermeladas a base del ovo. } \\
\text { - El pueblo negro afroecuatoriano de } \\
\text { Carpuela, Mascarilla y Apaquí utiliza la } \\
\text { arcilla para artesanías como máscaras con } \\
\text { rostros afro, estatuas, candelabros, porta } \\
\text { inciensos y nacimientos, artículos } \\
\text { decorativos y utilitarios. } \\
\text { En el Carnaval Coangue se realizan una } \\
\text { serie de actividades como el festival de } \\
\text { música y danza, exposición y venta de } \\
\text { artesanías, venta de comida típica, coplas, } \\
\text { poesía y actividades deportivas. }\end{array}$ \\
\hline $\begin{array}{l}\text { Runa Tupari } \\
\text { Native Travel } \\
\text { que en quíchua } \\
\text { significa } \\
\text { "Encuentro con } \\
\text { Indígenas". }\end{array}$ & $\begin{array}{l}\text { El turista experimenta un } \\
\text { contacto directo con la } \\
\text { naturaleza y, sobre todo se } \\
\text { enriquecerse de una } \\
\text { vivencia intercultural, } \\
\text { compartiendo múltiples } \\
\text { actividades con las familias } \\
\text { indígenas. }\end{array}$ & $\begin{array}{l}\text { - Atractivos ecológicos, recreativos y } \\
\text { culturales, como festividades indígenas, } \\
\text { artesanías y artes populares, y un paisaje } \\
\text { esplendoroso. } \\
\text { - La Reserva Ecológica Cotacachi-Cayapas: } \\
\text { parque natural de } 204.000 \text { ha, con clima es } \\
\text { frío en la zona alta, caluroso y húmedo en } \\
\text { la zona baja tropical, gran biodiversidad. } \\
\text { - El lago Cuicocha: antiguo cráter volcánico, } \\
\text { de más de } 200 \text { m, con } 2 \text { domos volcánicos, } \\
\text { aguas cristalinas, toma coloraciones azul } \\
\text { marino, verde esmeralda y la gama del arco } \\
\text { iris. } \\
\text { El volcán Cotacachi: de cresta nevada tiene } \\
\text { paredes empinadas y una magnífica vista } \\
\text { del cráter. La zona cuenta con lagos y una } \\
\text { diversidad de flora y fauna. }\end{array}$ \\
\hline
\end{tabular}




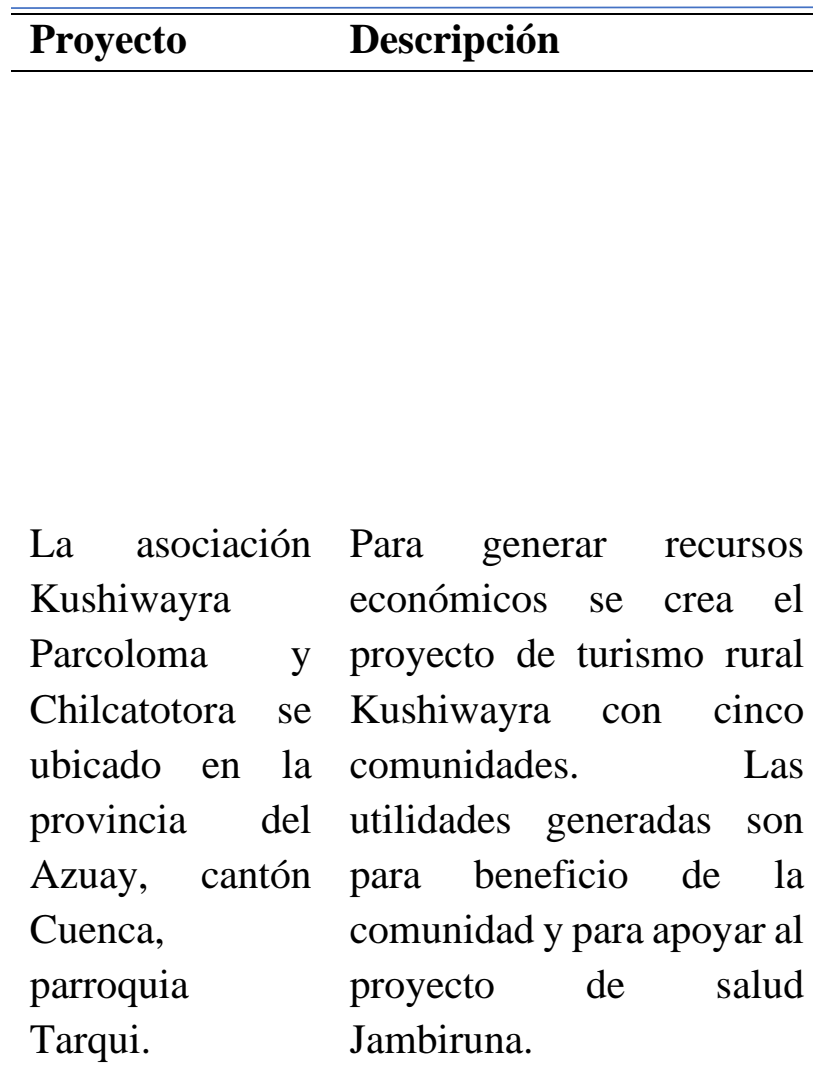

Características

- Cotacachi: expresiones culturales y artesanos trabajan el cuero, las comunidades indígenas muestran tradiciones. Música, danza, festividades, rituales y un exquisito arte culinario.

- 12 alojamientos rurales en diferentes comunidades indígenas del sector rural del Cantón Cotacachi.

- Paisaje andino, plantas medicinales, miradores, sendero dentro del cual se observa el Ingacuca, Chapanaloma, Jambiñan, plantas medicinales, área de recreación con su mirador Añaspungo, Camino del Inka, cabaña de Atuhuaico, sendero de Sigsahuaitiana.

- Camino del Inka, actividades agrícolas, producción de quesos, grupos de danza, comunidades de Chilca, Totora y Parcoloma, que trabajan en un solo producto; el grupo de danza de Chilcatotora, el grupo de música andina de las dos comunidades; cabaña Ayllu Huasi, en la pampa de Sigsahuaitiana; comida típica del lugar, tejidos en prendas de lana y de la agroindustria quesos.

- Caminata por la ruta de Parcoloma.

- Actividades agrícolas, como el trasquile de lana de borrego, asado de cuyes, caminatas a caballo, hilado de lana de borrego, molido de piedra.

- Degustación de las bebidas típicas en maíz, trigo y quinua en los refrigerios.

- Recorrido por la comunidad en los huertos de plantas medicinales.

Asociación de Se ubica en la provincia del

- Páramo, paisaje andino, laguna Culebrillas, Turismo Cañar, cantón Cañar, Comunitario parroquia Ingapirca, en las Ally Tuta Manta comunidades de Kaguanapamba y Sisad. cima del Yanacauri, reservorio en el hato de alpacas.

- Comunidades trabajan por el desarrollo de un producto turístico 


\begin{tabular}{|c|c|c|}
\hline Proyecto & Descripción & Características \\
\hline $\begin{array}{l}\text { Caguanapamba } \\
\text { y Sisid }\end{array}$ & $\begin{array}{l}\text { Turismo con gestión local } \\
\text { como una actividad } \\
\text { productiva, alternativa y } \\
\text { revalorizadora del } \\
\text { patrimonio sociocultural y } \\
\text { natural de las comunidades } \\
\text { gestoras, cuyo propósito es } \\
\text { mejorar la calidad de vida } \\
\text { de sus poblaciones }\end{array}$ & $\begin{array}{l}\text { - Visita a tiendas de artesanías en fibra de } \\
\text { alpaca y de la agroindustria, como frutillas } \\
\text { y plantas medicinales. } \\
\text { - Caminata, recorrido y visita al hato de } \\
\text { alpacas. } \\
\text { - Visita al taller de mujeres tejedoras } \\
\text { Galway, que trabajan en fibra de alpaca y } \\
\text { lana de borrego. } \\
\text { - Degustación de las bebidas típicas en maíz, } \\
\text { trigo y quinua. } \\
\text { Recorrido por la comunidad en los huertos } \\
\text { de plantas medicinales y huertos de } \\
\text { frutillas, en un sistema de granja integral } \\
\text { agroecológica. }\end{array}$ \\
\hline $\begin{array}{l}\text { Carbonería. } \\
\text { Comunidad } \\
\text { Shayacrrumi }\end{array}$ & $\begin{array}{lrr}\text { Generar y } & \text { dar valor a sus } \\
\text { recursos } & \text { naturales y } \\
\text { culturales } & \text { que } & \text { presentan } \\
\text { atractivos } & & \text { agrícolas, } \\
\text { pecuarios y } & \text { forestales, } \\
\text { como el } & \text { bosque } \\
\text { Carbonería. } & \end{array}$ & $\begin{array}{l}\text { - Paisaje andino, miradores, el bosque } \\
\text { Carbonería, granjas agrícolas donde se } \\
\text { realizan actividades de pesca, agricultura } \\
\text { tradicional cañari, criadero de animales } \\
\text { menores. } \\
\text { - La comunidad Shayacrrumi, que cuenta } \\
\text { con } 30 \text { familias que se dedican a la } \\
\text { actividad turística. } \\
\text { - Caminata por el bosque de Carbonería, } \\
\text { donde existen algunos puntos estratégicos } \\
\text { como miradores y lugares sagrados para } \\
\text { rituales. } \\
\text { Actividades de sanación del cuerpo, ritos y } \\
\text { temascales en el bosque Carbonería. } \\
\text { Estudios de plantas y de semillas nativas. } \\
\text { Observación de pájaros como cóndores, } \\
\text { pugas, tórtolas, yutes (perdices), } \\
\text { carpintero, gaviotas; y animales silvestres } \\
\text { como venados, conejos, cóndores. } \\
\text { Producción de llamas, se podrá montar } \\
\text { llamas, tomar fotografías y observar el } \\
\text { tejido de algunos artículos hechos en lana. } \\
\text { Visita a miradores, Taita Chavar, } \\
\text { Arrayanpamba y Lamaypata. }\end{array}$ \\
\hline
\end{tabular}




\begin{tabular}{|c|c|c|}
\hline Proyecto & Descripción & Características \\
\hline Asociación & La comunidad Pulinguí & - El Chimborazo, nevado perpetuo que \\
\hline Turismo & San Pablo, y su Centro de & alcanza los $6.310 \mathrm{msnm}$, con tres cumbres \\
\hline Comunitario & Turismo Comunitario Casa & glaciares, dotado de refugios y cargado de \\
\hline Cóndor & $\begin{array}{lll}\text { Cóndor, se ubica al } \\
\text { noroeste de la ciudad de }\end{array}$ & $\begin{array}{l}\text { leyendas y tradiciones para los pueblos que } \\
\text { se asientan a su alrededor. }\end{array}$ \\
\hline & $\begin{array}{l}\text { Riobamba, a } 37 \mathrm{~km} \text { en la } \\
\text { vía a los Refugios del } \\
\text { nevado Chimborazo, } \\
\text { parroquia San Juan, cantón }\end{array}$ & $\begin{array}{l}\text { - Convivencia con la acogedora y } \\
\text { emprendedora comunidad, dispuesta a } \\
\text { compartir su rica cultura: gastronomía, } \\
\text { música y danza, tradiciones y leyendas. }\end{array}$ \\
\hline & $\begin{array}{l}\text { Riobamba, } \quad \text { provincia } \\
\text { Chimborazo. }\end{array}$ & $\begin{array}{l}\text { - Lugar tranquilo para el descanso y la } \\
\text { aventura, en cabañas, albergues, casas de } \\
\text { familia, que proporcionan alojamiento con } \\
\text { dormitorios de cuatro, } 10 \text { o } 14 \text { camas, para } \\
\text { grupos de hasta } 35 \text { personas. }\end{array}$ \\
\hline Fundación & Enclavada en las praderas & - El volcán Cotopaxi y los Illinizas \\
\hline Ecuatoriana & del Taita Illiniza, se ubica & constituyen los atractivos más importantes. \\
\hline Pastocalle. & $\begin{array}{l}\text { la parroquia Pastocalle, al } \\
\text { noreste de la capital } \\
\text { provincial Latacunga, } \\
\text { cantón } \\
\text { provincia de Cotopaxi. }\end{array}$ & $\begin{array}{l}\text { - La cascada de Cunuyacu, conformada por } \\
\text { las aguas termales que salen de la montaña. } \\
\text { - Atractivos naturales tales como la laguna } \\
\text { de los Patos y el bosque de La Aliaga. } \\
\text { - El servicio de alimentación oferta platos } \\
\text { típicos de la zona. }\end{array}$ \\
\hline
\end{tabular}

Fuente: Elaboración propia con aportes de (González, 2008)

En Ecuador la evidencia empírica de gestión de turismo rural se da en numerosas circunscripciones territoriales, como los mostrados anteriormente. Este turismo rural tiene la característica de ser un turismo deseado y controlado por la gente de la localidad que lo desarrolla. $\mathrm{Su}$ fortaleza reside en los aspectos relacionados con las capacidades del habitante rural para relacionarse con los turistas por medio de palabras, actitudes, el entorno y las actividades propias del medio rural. Tal como lo describe Christaller (1966) "la valoración de los atractivos, permite el diseño de rutas turísticas (productos turísticos), teniendo en cuenta su optimización, ubicación y homogeneidad socio cultural, aspectos señalados por el modelo centro periferia"

En Ecuador también existen haciendas, fincas y ranchos que son un modelo de actividades agro turísticas sostenibles, que combinan lo natural, con la gastronomía y la cultura. El Gobierno Provincial del Guayas creó seis rutas turísticas para fortalecer la actividad del turismo rural desarrollado en haciendas, fincas, granjas para que sean conocidos y valorados, las mismas se muestran a continuación: 
Cuadro 3. Rutas y Estructuras Agro turísticas en Ecuador

\begin{tabular}{|c|c|c|}
\hline Rutas & Cantones & Hacienda/finca/rancho agro turísticas \\
\hline $\begin{array}{l}\text { Ruta del } \\
\text { Arroz }\end{array}$ & $\begin{array}{l}\text { Lomas de Sargentillo, Isidro } \\
\text { Ayora, Pedro Carbo, Palestina, } \\
\text { Colimes, Balzar, Salitre y Daule. }\end{array}$ & - Hacienda las Habras-Colimes \\
\hline $\begin{array}{l}\text { Ruta del } \\
\text { Cacao }\end{array}$ & Naranjal y Balao & $\begin{array}{l}\text { - Hacienda Las Cañas- Naranjal, Recinto Puerto Inca. } \\
\text { - Hacienda Jambelí-Naranjal, recinto Balao Chico }\end{array}$ \\
\hline $\begin{array}{l}\text { Ruta del } \\
\text { Azúcar }\end{array}$ & $\begin{array}{l}\text { Jujan, Simón Bolívar Milagro, } \\
\text { Naranjito, Marcelino Maridueña y } \\
\text { Bucay }\end{array}$ & $\begin{array}{l}\text { - Hacienda La Danesa - Naranjito Km } 26 . \\
\text { - Rancho Vasija de Barro- Milagro, recinto El Recreo } \\
\text { - Finca El Chaparral - Milagro, Parroquia Roberto } \\
\text { Astudillo. }\end{array}$ \\
\hline $\begin{array}{l}\text { Ruta del } \\
\text { Pescador }\end{array}$ & $\begin{array}{l}\text { Playas, Guayaquil, Progreso } \\
\text { Posorja, Puerto el Morro y Puná }\end{array}$ & $\begin{array}{l}\text { - Hacienda El Castillo - Guayaquil km } 8 \text { vía a la Costa } \\
\text { - Finca La Gloria - Guayaquil, km 51, vía a la costa. } \\
\text { - Rancho Rio Chico - Guayaquil, Km } 51 \text { vía a Playa. }\end{array}$ \\
\hline $\begin{array}{l}\text { Ruta de la } \\
\text { Aventura }\end{array}$ & Bucay, El Triunfo y Elempalme & $\begin{array}{l}\text { - Hacienda Mundo San Rafael Bucay, Km } 8.5 \text { Vía } \\
\text { Naranjito Bucay } \\
\text { - Hacienda turística El Cadeal - Km 13.5 Vía Bucay, } \\
\text { Bosque la Esperanza. } \\
\text { - Refugio Campestre La Palma Azul - El Triunfo, } \\
\text { Recinto el Piedrero. } \\
\text { - Rancho el Borinquen - El Empalme, Parroquia El } \\
\text { Rosario } \\
\text { - Hacienda Bella Marina - El Empalme, Recinto Santa } \\
\text { Lucia }\end{array}$ \\
\hline $\begin{array}{l}\text { Ruta de la } \\
\text { FE }\end{array}$ & $\begin{array}{l}\text { Durán, Yaguachi, Nobol, Dauley } \\
\text { Guayaquil }\end{array}$ & $\begin{array}{l}\text { - Rancho DE NOS, Nobol, Km } 33 \text { vía a Daule. } \\
\text { - Asociación de Cacaoteros La Unión - San Jacinto de } \\
\text { Yaguachi. } \\
\text { - Rancho Olguita - San Jacinto de Yaguachi. }\end{array}$ \\
\hline
\end{tabular}

Fuente: Elaboración propia con aportes de (Zambrano, Zambrano, Mieles, \& Castelo, 2017) y el Gobierno Provincial del Guayas. 
Dentro de las actividades más demandadas en la actualidad se encuentran las visitas a las comunidades indígenas que la práctica de deportes y la observación de la flora y la fauna. Es decir, las actividades más solicitadas son las que tienen una estrecha relación con el turismo rural.

El turismo rural comunitario, como concepto que promueve el desarrollo, se ha posicionado políticamente en Ecuador; sin embargo, a pesar de que la organización comunitaria y gestión de sus habitantes y etnias, actualmente asociados en La Federación Plurinacional de Turismo Comunitario del Ecuador (FEPTCE), y al respaldo institucional al turismo rural comunitario como uno de los proyectos contemplados en el Plan Estratégico de Desarrollo Turístico Sostenible del Ecuador, PLANDETUR 2020, instrumento de planificación planteado y formulado de modo inclusivo y participativo por el Ministerio de Turismo de Ecuador, queda todavía mucho por hacer, muchas comunidades en territorios rurales necesitan no solo de una acertada gestión turística, también es necesario realizar estudios profundos cuyo objeto de estudio ha de aportar interpretaciones que enriquezcan la teoría y la práctica a favor de un desarrollo diferente, pues en la mayoría de territorios rurales, especialmente en las comunidades indígenas está todavía presente la inequidad, la enajenación, la exclusión con presencia de altos niveles de pobreza.

Adicionalmente a lo señalado, la realidad es que si bien la actividad turística ecuatoriana es considerada como área estratégica para el crecimiento de la economía que tiene una practicidad positiva en diferentes territorios; sin embargo, todavía es insípida, pues no ha logrado establecer planes adecuados integrales y sostenibles de desarrollo, debido a restricciones presupuestarias, inestabilidad política, escasa secuencia en la gestión de los organismos oficiales de turismo, falta de consensos y cooperación entre los gremios, y su relación con el ámbito oficial 1 (PLANDETUR 2020, 2012).

Las zonas rurales se caracterizan por permitir un desarrollo empresarial turístico de fácil acceso con altos niveles de crecimiento y rentabilidad. Se ha constatado la escasez o ausencia de estudios relativos a la empresa turística rural del Ecuador. La relativa novedad en cuanto al nacimiento de esta nueva tendencia en la oferta turística ha generado interés por parte del estado y de las comunidades que potencialmente serían beneficiadas.

Los atractivos turísticos son el principal motivador para que el turista ingresé al Ecuador, dada su naturaleza, cultura, geografía y su gente le ofrece un gran potencial de ser un atractivo turístico muy visitado. El turismo rural sostenible, organizado constituye una oportunidad para que las comunidades se integren a la actividad turística dentro del sistema productivo, para que esto suceda se debe analizar el entorno los atractivos y los recursos del que disponen y establecer los mecanismos de planificación que sean adecuados para el potencial turístico de la zona. Es necesaria la unificación de los organismos tanto públicos como privados y las comunidades de los sectores a fin de proponer y mejorar un desarrollo rural sostenible del turismo. 
En este contexto y siendo consecuentes con las dimensiones del turismo rural anteriormente planteados es necesaria la participación de las comunidades y de los beneficios que generen las actividades turísticas con el fin de mejorar las condiciones de vida y de trabajo de los habitantes de las zonas rurales que cuentan con la posibilidad de recibir visitantes, actividad que debe ser planificada, ejecutada y evaluada bajo un contexto de sostenibilidad.

\section{Conclusiones}

- El turismo rural se constituye en una herramienta de desarrollo de comunidades que cuentan con este potencial y están en capacidad de competir con otros atractivos, articula recursos naturales y culturales, historia, identidad y autenticidad.

- Siendo el turismo rural una actividad de carácter territorial que se desarrolla como parte de una actividad comunitaria, donde se articula un producto turístico, es necesario para su ejecución en primera instancia el apoyo gubernamental y el compromiso de participación de las familias o de la comunidad.

- Ante el desafío de los diversos destinos turísticos para responder a la demanda de turistas que quieren romper con lo rutinario, se van impulsando en Latinoamérica iniciativas locales para incluir actividades de ocio, de esparcimiento y atractivos naturales a través de las actividades productivas locales, por lo que comunidades-destino realizan permanentes esfuerzos por mejorar su competitividad.

- A la carencia de estudios de investigación de esta actividad en el Ecuador y en general en los países en vías de desarrollo, es necesario enfocar estudios investigativos a partir de objetos de estudio que enriquezcan la teoría y la práctica a favor de un desarrollo diferente, por y para el hombre que evite la enajenación, el deterioro del medio ambiente, la inequidad y la exclusión de comunidades de sus propios territorios, la escasez de recursos naturales, la degradación, el deterioro de las regiones todo esto bajo un contexto de sostenibilidad como una de las estrategias más viables para corregir y potenciar los procesos de desarrollo.

\section{Referencias Bibliográficas}

Fundación Suiza de Cooperación para el Desarrollo. (2014). Conceptos básicos para la gestión de Destinos Turísticos. Recuperado de https://www.swisscontact.org/fileadmin/user_upload/COUNTRIES/Peru/Documents/Pub lications/Conceptos_basicos_para_la_gestion_de_destinos_turisticos.pdf. Lima-Perú: Swisscontact.

Ministerio de Comercio Exterior y Turismo. (2010). Lineamientos para el desarrollo rural en el Perú. Lima: Ed. del Ministerio de Comercio Exterior y Turismo. Recuperado el 2016 
Aguirre, P., Blanco, D., \& Solís, E. (2010). Gestión Integral Turística Rural Sostenible: aplicada al municipio de Tamanique, Departamento de laLibertad. Obtenido de http://ri.ues.edu.sv/1853/1/Gesti\%C3\%B3n_integral_tur\%C3\%ADstica

Alcívar-Vera, I., \& Bravo-Acosta, O. (2017). Turismo sostenible: una alternativa de desarrollo comunitario desde un componente cultural. Espirales Revista Multidisciplinaria de $\begin{array}{llllll}\text { investigación } & \text { No. } & 9 & \text { Octubre. } & \text { recuperado de }\end{array}$ http://www.revistaespirales.com/index.php/es/article/view/114/60, 2-13.

Arteaga-Vera, J. (2017). MODELO DE GESTIÓN SUSTENTABLE (ECONÓMICO,SOCIAL Y AMBIENTAL) PARA EL ECOTURISMO EN LA PROVINCIA DE MANABÍ. Quito: Universidad de Especialidades Turísticas-UDET.

Ávila-Bercial, R., \& Barrado-Timón, D. (2005). NUEVAS TENDENCIAS EN EL DESARROLLO DE DESTINOS TURÍSTICOS: MARCOS CONCEPTUALES Y OPERATIVOS PARASU PLANIFICACIÓN Y GESTIÓN. Cuadernos de Turismo V15, $27-43$.

Barrado-Timón, D. (2004). EL CONCEPTO DE DESTINO TURÍSTICO. UNA APROXIMACIÓN GEOGRÁFICO-TERRITORIAL. Estudios Turísticos, $n \cdot{ }^{\circ} 160$. Recuperado de: http://estadisticas.tourspain.es/img-iet/Revistas/RET-160-2004-pag4568-91963.pdf, 45-68.

Barrera , E., \& Muñoz, R. (2003). Manual de Turismo Rural para Micro Pequeños y Medianos Empresarios Rurales. Buenos Aires: Ed. Promer.

Bigné, E. (2000). Marketing de destinos turísticos. Barcelona-España: ESIC Editorial.

Bligoo. (2016). http://destinosyproductosturisticosdelmundo.bligoo.com.mx/producto-turistico\#.

Bolwel , D., \& Weinz , W. (2009). Reducir la pobreza a través del turismo, Documento de trabajo, Organización Internacional del Trabajo. Ginebra.: OIT-ISBN: 978-92-2-321644-3.

Bosch,C; Marco,L y Cabado, L. (1998). Turismo y Medio ambiente. (C. d. Ramón, Ed.) Madrid, España. Recuperado el Octubre de 2017

Burns, P., \& Holden, A. (1995). "ourism: A new perspective. London: Ed. Prentice Hall.

Cabarcos, N. (2011). Administración de Servicios Turísticos. . Bogotá, Colombia: Ideas propias Editorial,.

Campbell, L. (1999). Ecotourism in Rural Developing Communities. Annals of Tourism Research, 26, 534-553.

CCM, E. (Febrero de 2016). Obtenido de Introducciòn a la calidad. Obtenido de http://es.ccm.net/contents/606-calidad. 
Checkland, P., \& Scholes, J. (1994). La metodología de sistemas suaves en acción. México: MEGABYTE y Limusa.

Cherifi, B., Smith, A., Maitland, R., Stevenson, N., MacCoun, R., Uriely, N., \& Comunello, F. (2014). Glossary of tourism terms. Annals of Tourism Research. Organización Mundial de Turismo (UNWTO). http://cf.cdn.unwto.org/sites/all/files/Glossary+of+terms.pdf.

Christaller, W. (1966). Central places in southem Germany. Germany: Prentice Hall.

Cisneros, G. G. (1990). Posicionamiento estratègico y gestiòn de marcas turìstica. Recuperado el 20 fe febrero de $2016, \quad$ de http://www.papersdeturisme.gva.es/ojs/index.php/Papers/article/viewFile/315/269

Combariza, J. (2012). El turismo rural como estrategia de desarrollo sostenible: caso municipio de La Mesa (Cundinamarca). Universidad Nacional de Colombia : Bogotá.

Cortés , J. (1996). Comunicación Interpretativa: variable clave en el Marketin Mix de las Empresas de Turismo Rural. Estudios Turísticos $n^{\circ}$ 130, 37-50.

Cuervo, R. (1967). El turismo como medio de comunicación humana. México: Departamento de Turismo.

De La Torre-Padilla, O. (1980). Turismo-fenómeno social. México: Fondo de Cultura Económica.

Decasper, S. (2014). Competitividad y desarrollo sostenible en el sector turístico. 1/8.

Durston, J. (2001). Capital Social, parte del problema, parte de la solución: su papel en la persistencia y en la superación de la pobreza en América Latina y el Caribe. Conferencia "En busca de un nuevo paradigma: capital social y reducción de la pobreza en América latina y el Caribe”. Santiago de Chile: CEPAL.

Dwyer, L y Kim Ch. (2003). Destination competitiveness. Determinants and Indicators (Vol. 6). Recuperado el 31 de Octubre de 2017

Dwyer, L., \& Kim, C. (2003). Destination Competitiveness Determinants and indicators. 369-414.

Dwyer, L., Forsyth, P., \& Rao, P. (2000). “The price competitiveness of travel and tourism: a comparison of 19 destinations”. Tourism Management.

Faría, C., Angarita, J. L., Pérez, L., \& Ochoa, A. (2016). Modelo de planificación estratégica del turismo rural sostenible. (U. A. México, Ed.) redalyc.

Flores-Ruiz, D. (2007). COMPETITIVIDAD SOSTENIBLE DE LOS ESPACIOS NATURALES PROTEGIDOS COMO DESTINOS TURÍSTICOS: UN ANÁLISIS COMPARATIVO DE LOS PARQUES NATURALES SIERRA DE ARACENA Y PICOS DE AROCHE Y SIERRAS DE CAZORLA, SEGURA Y LAS VILLAS. Huelva-España: Tesis Doctoral de la 
UNIVERSIDAD DE HUELVA-FACULTAD DE CIENCIAS EMPRESARIALESDEPARTAMENTO DE ECONOMÍA GENERAL Y ESTADÍSTICA.

Fuentes, A. (2013). Teoría y Desarrollo del Turismo en el Ecuador. Ecuador: Efecto Gráfico.

Garau, J. (2006). Propuesta de dos índices para la mediación de la competitividad de los destinos de sol y playa del Mediterráneo: avance de resultados desde el punto de vista de la demanda. XV Simposio Internacional de turismo y ocio,. Barcelona: ESADE-Fira.

García. (1998). Planes de desarrollo desde los municipios turísticos los planes de excelencia y de dinamización turística en La Gestión de la Calidad en el Municipio Turístico. Ponencias del IV Seminario del Foro Permanente Turismo y Municipio. Andalucia.

Gascón, J. y Cañada, E. (2005). Viajar a todo tren: Turismo, desarrollo y sostenibilidad. Barcelona:Icaria. Recuperado el 9 de Mayo de 2017

Gascón, R. (2011). Turismo rural comunitario y diferenciación campesina. Consideraciones a partir de un caso andino. Mundo Agrario, 11(22). doi:ISSN 1515-5994

Genest, J., \& Legg, D. (2003). Premier-ranked Tourist Destinations: Development of a Framework for Analysis and its Self-guided Workbook. Obtenido de http://www.tourism.gov.on.ca/english/research/pdf/self-guided-workbook.pdf.

Goeldner, C: Ritchie J y McIntosh, R. (s.f.). Tourism: principles, practices, philosophies (8th ed.). 2000. (J. W. Inc., Ed.) Toronto, EUA. Recuperado el Octubre de 2017

Gomera, C. Y. (2016). http://www.ecoturismocanarias.com/gomera/estudio/11.htm.

Gomes, A. (2015). El uso del internet en la comunicación con los clientes. La tenciónon-line en los hoteles Florianopolis (Santa Catarina, Brasil). IELUSC, Joenville-Brasil.

González, M. E. (2008). Turismo Rural Ecuador . Ecuador: Instituto Interamericano de Cooperación para la Agricultura .

González-Ávila, M. (2011). Una propuesta para desarrollar turismo rural en los municipios de Zacatecas, México: las rutas agro-culturales. PASOS. Revista de Turismo y Patrimonio $\begin{array}{lllllll}\text { Cultural } & \text { Vol. } & 9 & N^{o} & 1 . & \text { Recuperado de }\end{array}$ http://www.pasosonline.org/Publicados/9111/PS0111_11.pdf.

Gooroochurin, N y Sugiyarto, G. (2004). Mediendo la Competitividad en los viajes de la Industria y el Turismo. (N. U. School, Ed.) Recuperado el Octubre de 2017

Hamilton, C. (2006). El Fetiche del Crecimiento. Pamplona-España: Ed. Laetoli.

Herrero, H. (2006). Los paradigmas de la sustentabilidad. ¿ Hacia una revolución ética y solidaria? Desarrollo Sostenible, 25. Recuperado el 20 de Octubre de 2017 
Hickman, L. (2007). The final call: In search of the true cost of our holidays”. London: Eden Projects Books \& GBM.

Inostroza, G. (2008). Modelo de gestión sostenible del turismo comunitario en la región andina. Obtenido de redalyc: http://www.redalyc.org/html/2233/223314985006/

Jaime-Pastor, V., Casas-Jurado, C., \& Amparo-Soler, D. (2011). DESARROLLO RURAL A TRAVÉS DEL TURISMO COMUNITARIO. ANÁLISIS DEL VALLE Y CAÑÓN DE COLCA. Rev. Gestión Turística $N^{\circ}$ 15, Enero-junio, 01-20.

Jiménez-García, M. (2006). TURISMO Y UNIÓN EUROPEA: UNA PROPUESTA DE POLÍTICA COMUNITARIA Y DE INNOVACIÓN COMERCIAL EN EL MEDITERRÁNEO EUROPEO. Sevilla- España: UNIVERSIDAD DE SEVILLA-Departamento de Economía Aplicada.

Kayser, B. (1990). La renaissance rurale. Sociologie des campagnes du monde occidental. París: Armand Colin, París.

Kieffer, M. (2018). Turismo Rural Comunitario y organización colectiva: un enfoque comparativo en México. PASOS. Revista de Turismo y Patrimonio Cultural Vol. 16 N.o 2. https://doi.org/10.25145/j.pasos.2018.16.030, 429-441.

Lai, Jai. Ad-hoc. (2008). Travel and Tourism. an industry primer. PEARSON. Prentice Hall. Recuperado el 15 de Marzo de 2016, de ISBN 013-170129-0

Leiper, N. (1979). The framework of tourism: Towards a definition of tourism, tourist and the tourism industry. Annals of Tourism Research, vol. 4, $n^{\circ} 6$. Recupereado de https://www.sciencedirect.com/search? qs=tourism\%20industry\&authors $=$ Leiper $\% 2 \mathrm{C} \% 2$ ONeil\&pub=Annals\%20of\%20Tourism\%20Research\&volume $=4 \&$ issue $=6 \&$ page $=309$ $407 \&$ show $=25 \&$ sortBy $=$ relevance \&origin $=j r n l \_i s s u e \& z o n e=, 309-407$.

López, J. y Sánchez, S. (30 de 02 de 2009). Desarrollo socieconómico de las zonas rurales con bases en el turismo comunitario. Cuadernos de desarrollo rural, 2009, 1/18. doi:ISSN:0122-1450

López-Cordonez , M. (2017). EL SERVICIO DE TURISMO COMO ALTERNATIVA SUSTENTABLE Y SU IMPACTO EN EL DESARROLLO COMUNITARIO EN LA PARROQUIA ULBA DEL CANTON BAÑOS DE AGUA SANTA DE LA PROVINCIA DE TUNGURAHUA DURANTE EL AÑO 2015-2016. Quito-Ecuador: UNIVERSIDAD TECNOLÓGICA INDOAMÉRICA-CENTRO DE ESTUDIOS DE POSTGRADOMAESTRÍA EN GESTIÓN DE PROYECTOS SOCIOPRODUCTIVOS.

Maldonado , C. (2005). Pautas metodológicas para el análisis de experiencias de turismo comunitario. Documento de trabajo $n^{o} 13$ de la Oficina Internacional de Trabajo. ISBN: 92-2-317378-7. Ginebra: OIT. 
Manyara , G., \& Jones , E. (2007). Community-based tourism enterprises development in Kenya: An exploration of their potential as avenues of poverty reduction. Journal of Sustainable Tourism, Vol. 15, ISNN: 1747-7646., 628-644.

Mara, R y Varzin, G. (2008). Modelos de Competitividad para destinos turísticos en el marco de la sostenibilidad.

Martìn, R. (2015). Principios, organizaciòn y pràctica del turismo. La Habana: Universitaria Fèlix Varela.

Martínez-Moreno, O., \& Ruiz-Andrade, J. (2009). Las particularidades de la agenda 21 para el turismo mexicano. Un análisis de la aplicación del sistema de indicadores de sustentabilidad en el municipio de Playas de Rosarito, Baja California. Gest. tur, $N^{\circ} 12$, Dic. ISSN 0717 - 1811, 09-29.

Ministerio de Turismo de Ecuador. (08 de junio de 2018). Cerca de un millón de extranjeros arribaron al país entre enero y mayo de 2018. Obtenido de Ministerio de Turismo: https://www.turismo.gob.ec/cerca-de-un-millon-de-extranjeros-arribaron-al-pais-entreenero-y-mayo-de-2018/

MINTUR. (2017). Boletín de Estadísticas Turísticas 2011-2015. Quito: Ministerio de Turismo del Ecuador .

Miranda-Andrade, R. (2018). MODELO DE GESTIÓN PARA CONVERTIR LA PLAYA DE LA TIÑOSA EN EL CANTÓN MANTA EN UN DESTINO TURÍSTICO ACCESIBLE PARA TODOS. Quito: UNIVERSIDAD DE ESPECIALIDADES TURÍSTICAS-MAESTRÍA EN GESTIÓN DEL TURISMO, Recuperado de: http://190.95.226.74/handle/45000/415.

Moller, R. (2010). PRINCIPIOS DE DESARROLlO SOSTENIBLE PARA AMÉRICA LATINA. Rev. Ingeniería de Recursos Naturales y del Ambiente, núm. 9, enero-diciembre Recuperado de: http://www.redalyc.org/articulo.oa?id=231116434012, 101-110.

Montesdeoca, C., \& Vera, J. (2014). Plan de gestión integrada en el turismo rural del cantón Bolivar: Caso Parroquia Membrillo. Calceta : Escuela Superioir Politecnica de Manabi "Manuel Félix López".

Mora,M; Bernal,J; Paneso,J. (2016). Anatomía quirúrgica del ojo: Revisión anatómica del ojo. Morfolia, 24.

Mowforth, M., \& Munt, I. (1998). "Tourism and Sustainability: New tourism in the Thrid World". . London; New York: Ed. Routlledge.

Muñiz, R. (2016). Marketing XXI. Obtenido de la marca. Obtenido de http://www.marketingXXI.com 
Musa, F. (2001). Uma visão de conjunto. CLUSTER. Revista Brasileira de Competitividade, (Edição Especial), 194-207.

Network, W. T. (15 de Febrero de 2016). El Turismo y atenuación de la pobreza.

Nowicka, P, P. (2008). Vacaciones en el paraiso, turismo y desarrollo. Barcelona-España: Ed. Intermom OXFAM.

OMT. (2002). Manual on Tourism and poverty alleviation. Madrid: OMT, Madrid. ISBN: 97892-844-1343-0.

OMT. (2017). Tourism - an economic and social phenomenon. Obtenido de Why tourism?: http://www2.unwto.org/content/why-tourism

OMT. (25 de Junio de 2018). El turismo internacional supera las expectativas en los primeros meses de 2018. Obtenido de Press Release: http://media.unwto.org/es/press-release/201806-25/el-turismo-internacional-supera-las-expectativas-en-los-primeros-meses-de-2

OMT. (2018). Entender el turismo: Glosario Básico. Obtenido de UNWTO Home: http://media.unwto.org/es/content/entender-el-turismo-glosario-basico

ONU. (2013). Sustainable tourism: Contribution to economic growth and sustainable development, Expert Meeting of Tourism's Contribution to Sustainable Development. Genova: UNCTAD.

Parra-Cárdenas , A., Cevallos-Ortega,, F., \& Cisneros-Mustelier , L. (2018). Posicionamiento y sostenibilidad, contribución a la competitividad de destinos turísticos. Revista Investigaciones Sociales, Marzo Vol.4 No.11. Recuperado de http://www.ecorfan.org/republicofnicaragua/researchjournal/investigacionessociales/jou rnal/vol4num11/Revista_Investigaciones_Sociales_V4_N11_5.pdf, 33-45.

Pérez, L. (2004). Turismo Comunitario y Generación de Riqueza en países en vías de desarrollo. Un estudio de Caso en el Salvador. REVESCO, no. 99, 85-103.

Perez, R. (2006). Modelo y Procedimiento para la gesdtión de la calidad del destino turístico holguinero. 16/241. Holguín, Cuba. Recuperado el 1 de Octubre de 2017

PLANDETUR 2020. (2012). Plan estratégico de desarrollo de turismo sostenible para Ecuador . Quito: Ministerio del Turismo.

Porter, M. (1993). La ventaja competitiva de las naciones. Barcelona: Plaza y Janes Editores .

Prieto, M. (2011). Los estudios sobre turismo en Ecuador. Espacios en disputa. Quito: FLACSO, Sede Ecuador.

Ritchie, J y Crouch G. (2003). The competitive destination. (W. I. Publishing, Ed.) Recuperado el Octubre de 2017 
RITCHIE, J., \& CROUCH , G. (2003). The Competitive Destination. A Sustainable tourism perspective. CABI Publishing UK.

Rodrìguez, M. (2015). Obtenido de http://www.eumed.net/libros gratis

Rumipamba G. A. (2012). ): "Plan de Desarrollo y Ordenamiento Territorial de la Parroquia Rumipamba (2012-2025)”. . Sangolquí-Pichincha: GAR .

Sancho-Pérez, A., \& García-Mesanat, G. (2015). El posicionamiento de un destino turìstico en un mercado globalizado y competitivo: Comparación de prácticas entre competidores y líderes. Obtenido de http://www.uv.es/ sancho/benchmarking.pdf

Sancho-Pérez, García-Mesanat, G., Bueno, A., \& Yagüe-Perales, R. (2001). Auditoría de sostenibilidad en los destinos turísticos. Valencia: MININ.

Santamaría, E., \& Bayas, F. (2018). Efecto económico de la actividad turística en la provincia de Tungurahua, Ecuador. Ciencias Administrativas, Año 6 (11) Enero-Junio. Recuperado de https://revistas.unlp.edu.ar/CADM/article/view/3788/4099, 15-25.

SERNATUR. (2008). Orientaciones para el Diseño de un Plan de Desarrollo Turístico en Destinos Turísticos (PLADETUR) Chile. Obtenido de http://www.plataformacaldera.cl/biblioteca/589

Stronza, A., \& Gordillo, J. (2008). Community views of ecotourism. Annals of Tourism Research, $35,448-468$.

Toledo, G; Valdéz J y Pollero, A. (1998). Empresas turísticas en ambiente globalizado: marketing y competitividad. Estudio de casos de clusters turísticos. (FEA/USP, Ed.) Sao Paulo, Brasil. Recuperado el Octubre de 2017

Toselli, C. (2003). Turismo cultura, participación local y sustentabilidad. Algunas consideraciones sobre la puesta en valor del patrimonio rural como recurso turístico en Argentina. Obtenido de Portal Iberoamericano de Gestión Cultural: www.gestioncultural.org

Trivelli, C, Escobal, J y Revesz, B. (2009). Desarrollo rural en la sierra: aportes para el debate. Lima, Perù. doi:Cipca, Grade, IEP, Cies

UNESCO. (2012). Educación para el Desarrollo Sostenible. Organización de las Naciones Unidas para la Educación, la Ciencia y la Cultura . París : Sector Educación de la UNESCO.

Valls, J. (1998). Cambio de Concepto en la Gestiòn Turìstica del Municipio. Estudios de Gestiòn Turìstica, 7, 3-7. Recuperado el 19 de mayo de 2006

Valls, J. (2004). Gestión de destinos turísticos sostenibles. Barcelona-España: Ediciones Global 2000. 
Valls-Gimenez, J. (2007). Gestión de destinos turísticos sostenibles Primera Edición. Barcelona: Editorial: Gestión 2000.

Varisco, C. (2007). Iniciativas de desarrollo local y fomento del cl’puster turìstico. Aportes y transferencias. 11, 31-42.

Varisco, C. (2016). Turismo Rural: Propuesta Metodológica para un Enfoque Sistémico. PASOS. Revista de Turismo y Patrimonio Cultural Vol. 14 N.o 1, 153-167.

Vera, J., Palomeque, F., Marchena, M., \& Antón, S. (1997). Análisis Territorial del Turismo. Barcelona: Ed. Ariel.

Vilches, A., \& Gil-Pérez, D. (2016). La Ciencia de la Sostenibilidad: una necesaria revolución científica. Ciênc. Educ., Bauru, v. 22, n. 1,doi: http://dx.doi.org/10.1590/1516731320160010001, 1-6.

Viloria-Cedeño , N. (2012). DESARROLLO TURÍSTICO Y SU RELACIÓN CON EL TRANSPORTE. Gestión Turística, núm. 17, enero-junio. Recuperado de http://www.redalyc.org/articulo.oa? $i d=223324204002,23-36$.

Virgen-Aguilar, C., Hernández-Nogueda, V., \& González-González, M. (2016). La promoción y la publicidad como estrategia en la gestión de destinos turísticos. El caso Puerto Vallarta. Guadalajara: Universidad de Guadalajara, Centro Universitario de la Costa-Secretaría de Turismo del estado de Jalisco.

Von Bertalanffy, L. (1976). Teoría general de los sistemas. Fundamentos, desarrollo, aplicaciones. México: Fondo de Cultura Económica.

WWF International. (2001). Directrices para el desarrollo de turismo comunitario. Suiza: Editadopor WWF INTERNATIONAL.

Zambrano, J., Zambrano, R., Mieles, D., \& Castelo, J. (2017). Las haciendas agro-turísticas como medio de desarrollo del turismo rural en el Cantón Milagro, Ecuador. Revista Ciencia UNEMI.

Zizumbo-Villareal, L. (2013). Las paradojas del desarrollo local y del turismo. Ciudad de México: Universidad Autónoma del Estado de México, Miguel Ángel Porrúa.

Zurita, M., Román, G., Fonseca, G., Quevedo, L., \& Castillo, D. (2017). Turismo Rural Comunitario, Una Alternativa Para El Desarrollo De Los Territorios Rurales Estudio De Caso: Guasuntos, Ecuador. European Scientific Journal Jun, edition Vol.13, No.17. recuperado de: http://eujournal.org/index.php/esj/article/view/9513/9021, 40-53.

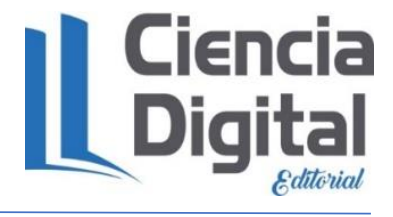




\section{Para citar el artículo indexado.}

Parra Cárdenas, A. V., Cisneros Mustelier, L., \& Velasteguí López, E. (2019). El turismo rural y aportaciones al desarrollo de comunidades en territorios locales. Explorador Digital, 3(4), 6-28. https://doi.org/10.33262/exploradordigital.v3i4.912

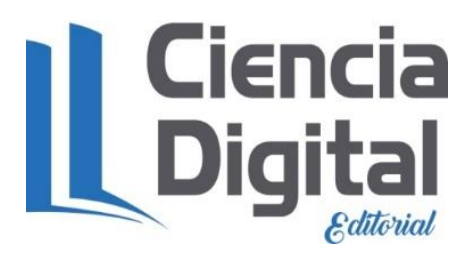

El artículo que se publica es de exclusiva responsabilidad de los autores y no necesariamente reflejan el pensamiento de la Revista Explorador Digital.

El articulo queda en propiedad de la revista y, por tanto, su publicación parcial y/o total en otro medio tiene que ser autorizado por el director o editor de la Revista Explorador Digital.

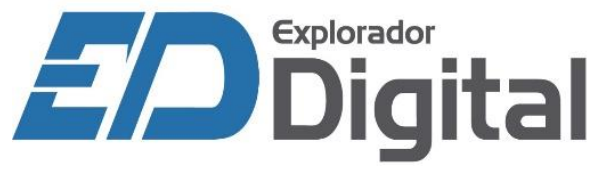

